\title{
Antituberculous Drugs
}

\author{
Jeeson C Unni
}

\section{ABSTRACt}

The RNTCP guidelines for management of pediatric tuberculosis is continuously evolving and this article highlights the 2019 recommendations:

- The treatment categories have been simplified to two; new and previously treated.

- The definitions of the types of drug resistance have been elucidated.

- Daily therapy is recommended as the treatment of choice.

- The continuation phase now includes three drugs; isoniazid, rifampicin and ethambutol as isoniazid monoresistance is around $13 \%$.

- The antituberculous therapy (ATT) dosing in children is higher as the pharmacokinetic studies have shown that children traditionally achieve lower serum levels than adults.

- Empiric category 2 regimen with extension of IP by 2 months has been withdrawn. It is now recommended to investigate for drug resistant TB (DR TB) in such cases.

- The indications for steroid usage and dosage in pediatric TB have been clearly defined. The evidence for the routine use of steroids in tuberculomas is unclear as of date.

- Pyridoxine supplementation is routinely recommended in children isoniazid is higher for both prophylaxis and treatment.

Keywords: Antituberculous drugs, BCG adenitis, Children, Tuberculosis.

Pediatric Infectious Disease (2019): 10.5005/jp-journals-10081-1109

\section{INTRODUCTION}

$T^{\circ}$ describe the recommendations for treatment of tuberculosis (TB), the latest RNTCP guidelines divide tuberculosis into two categories.

\section{Category I-New Case}

- A newly diagnosed case of TB that has never received antitubercular treatment (ATT) (Table 1) or has received ATT for less than 30 days.

\section{Category II-Previously Treated Case}

- Recurrent TB case-An AFB positive TB detected in a child who had been cured earlier with a full recommended course of ATT

- Treatment failure case-Treatment of cases where the most recent course of ATT had failed to cure the child.

- Treatment of cases lost to follow-up-An AFB positive TB detected in a child who had ATT for a month or more of their most recent course and had since been lost to follow-up

- Other previously treated case-Cases of TB where the outcome after their most recent course of treatment is unknown or undocumented

\section{Classification on the Basis of Drug Resistance}

- Monoresistance (MR): A TB patient whose biological specimen is resistant to one first-line anti-TB drug (ATD) only.

- Poly resistance (PDR): A TB patient whose biological specimen is resistant to more than one first-line ATD, other than both INH and rifampicin.

- Multidrug resistance (MDR): A TB patient whose biological specimen is resistant to both INH and rifampicin with or without the resistance of other first-line ATD, based on results from a quality assured laboratory.

- Rifampicin resistance (RR): Resistance to rifampicin detected by phenotypic or genotypic methods with or without resistant to
Editor-in-chief, Associate Senior Consultant

IAP Drug Formulary, Aster Children's Hospital, Aster Medcity, Kochi, Kerala, India

Corresponding Author: Jeeson C Unni, Editor-in-chief, Associate Senior Consultant, IAP Drug Formulary; Aster Children's Hospital, Aster Medcity, Kochi, Kerala, India, e-mail: jeeson1955@gmail.com

How to cite this article: Unni JC. Antituberculous Drugs. Pediatr Inf Dis 2019;1(1):37-38.

Source of support: Nil

Conflict of interest: None

\begin{tabular}{ll}
\hline \hline \multicolumn{2}{c}{ Table 1: IAP RNTCP 2019 classification } \\
\hline Type of patient
\end{tabular}

New AFB+ve pulmonary TB

New clinically diagnosed pulmonary TB

New clinically diagnosed rifampicin sensitive extrapulmonary TB

$2 H R Z E+4 H R E^{b}$

Drug-sensitive previously treated $\mathrm{TB}^{\mathrm{C}}$

(recurrence, treatment after loss to follow-up, treatment after failure)

${ }^{a}$ Molecular testing shall be done in all new cases in children with suspected $\mathrm{TB}$ at diagnosis.

${ }^{\mathrm{b}}$ In the case of neuro and spinal TB, the continuation phase is extended to 8 months

'All these categories of children shall be evaluated as DR TB suspects and evaluated as per DRTB algorithm. DST based treatment shall be followed. In case they are found to be drug-sensitive they shall be started on the above regimen as for a new case.

other ATD excluding INH. Patient with RR should be managed as if they are in MDR TB case.

- Extensive drug resistance (XDR): MDR TB case whose biological specimen was resistant to a fluoroquinolone (FQ) and a second-line injectable ATD from a quality assured laboratory.

(O) The Author(s). 2019 Open Access This article is distributed under the terms of the Creative Commons Attribution 4.0 International License (https://creativecommons. org/licenses/by-nc/4.0/), which permits unrestricted use, distribution, and non-commercial reproduction in any medium, provided you give appropriate credit to the original author(s) and the source, provide a link to the Creative Commons license, and indicate if changes were made. The Creative Commons Public Domain Dedication waiver (http://creativecommons.org/publicdomain/zero/1.0/) applies to the data made available in this article, unless otherwise stated. 
Table 2: Revised dosages of antituberculosis drugs

\begin{tabular}{|c|c|c|c|}
\hline $\begin{array}{l}\text { Drugs } \\
\text { Isoniazid }\end{array}$ & $\begin{array}{l}\text { Doses } \mathrm{mg} / \mathrm{kg} / \mathrm{d} \\
10\end{array}$ & $\begin{array}{l}\text { Range (mg) } \\
7.5-15\end{array}$ & $\begin{array}{l}\text { Maximum dose per } \\
\text { day }(\mathrm{mg}) \\
300\end{array}$ \\
\hline Rifampicin & 15 & $10-20$ & 600 \\
\hline Pyrazinamide & 35 & $30-40$ & 2,000 \\
\hline Ethambutol & 20 & $15-25$ & 1,500 \\
\hline Streptomycin & 15 & $15-20$ & 1,000 \\
\hline
\end{tabular}

The latest information regarding the pharmacokinetics of ATT, the dose, range of dose and the maximum permitted dose of an ATD are given in Table 2 .

\section{Fixed-dose Combinations (FDCs)}

Two types of FDCs (Table 3) - that with three ATDs and another incorporating four ATDs are available under RNTCP. Safety, simplification of therapy, decrease in dosing errors, less chance of missing administration of a prescribed ATD and decrease in the incidence of drug-resistant mycobacterium TB; are proposed advantages of using FDCs in ATT. It also allows for ease of mass transport, supply, and distribution of ATD in the National TB programs. FDC tablets of good quality and with proven bioavailability of rifampicin and in appropriate dosing combination should be used in the treatment of TB.

There are two types of FDCs available under RNTCP:

1. Three drugs FDC DT (H 50, R 75, Z150) (10:15:30) for children, + non-DT Ethambutol 100

2. Four drugs FDC Adult (H 75, R 150, Z 400, E 275).

The therapy recommended as per body weight is detailed as: indications for the use of steroids in the treatment of TB
Table 3: Recommended drug dosages and FDC Pill a combination from 0 to 18 years

\begin{tabular}{ll}
\hline Weight range $(\mathrm{kg})$ & Dose \\
\hline $4-7$ & $1 \mathrm{P}+1 \mathrm{E}$ \\
$8-11$ & $2 \mathrm{P}+2 \mathrm{E}$ \\
$12-15$ & $3 \mathrm{P}+3 \mathrm{E}$ \\
$16-24$ & $4 \mathrm{P}+4 \mathrm{E}$ \\
$25-29$ & $3 \mathrm{P}+3 \mathrm{E}+1 \mathrm{~A}$ \\
$30-39$ & $2 \mathrm{P}+2 \mathrm{E}+2 \mathrm{~A}$ \\
\hline
\end{tabular}

- Tuberculosis-immune reconstitution inflammatory syndrome (TB-IRIS) severe life-threatening manifestations and its sequelae to relieve symptoms of the paradoxical inflammation

- TBM

- TB pericarditis

- Addison's disease

- Miliary TB

- TB uveitis

Steroids may be used in endobronchial TB, significant compression of airway, mediastinal compression syndrome, pleurisy and laryngeal TB with respiratory distress.

Good evidence for use of steroid in management of in tuberculoma is lacking.

- Dose-Prednisolone: $1-2 \mathrm{mg} / \mathrm{kg} /$ day or dexamethasone-0.6mg/ $\mathrm{kg}$ or it's equivalent may be given up to four weeks and then tapered over next 4 weeks.

- Pyridoxine: $10 \mathrm{mg} /$ day is recommended for all children receiving $\mathrm{INH}$. 